\title{
Boubaker Polynomials and Their Applications to Numerical Solution of Differential Equations
}

\author{
Tinggang Zhao ${ }^{1, ~ a ~ *, ~ Y o n g j u n ~} \mathrm{Li}^{2, \mathrm{~b}}$ \\ ${ }^{1}$ School of Mathematics, Lanzhou City University, Lanzhou,730070,P. R. China \\ 2 School of Mathematics, Lanzhou City University, Lanzhou,730070,P. R. China \\ a13669397938@163.com, bli_liyong120@163.com
}

Keywords: Boubaker polynomial, Boubaker-Legendre transform, BPES, Numerical solution.

Abstract. In this paper, Boubaker polynomials and the Boubaker polynomial expansion scheme (BPES) are discussed. We present a transform from Boubaker polynomial to Legendre orthogonal polynomials. By this transform, the spectral-type numerical method using Boubaker polynomial can be deduced for seeking numerical solution of differential equations.

\section{Introduction}

Orthogonal polynomials, including Chebyshev's, Legendre's, Laguerre's Jacobi's and Hermite's, are extensively studied [1-2] which take a big part of numerical analysis. A noted example is so-called spectral method[3-5]. Meanwhile, there exist other class of algebraic polynomials, non-orthogonal polynomials, which are scarcely concerned by researchers. Say, the Boubaker polynomials, which are non-orthogonal algebraic polynomial sequence[6-8]. In practical, the polynomial expansion methods, such as Boubaker polynomial expansion scheme(BPES), are extensively applied to seek both analytic and numerical solution of diverse differential equations including initial value and boundary value problems in spite of orthogonality(see [6-14] and references therein). However, the Boubaker polynomials had been used to become a controversy topic about 2009, which Wikipedia chose not to host an article on the subject of Boubaker polynomials[13]. It is worthy to contemplate the problems about the Boubaker polynomials.

In this paper, we recall the definitions of the Boubaker polynomials, their properties, the BPES, and its applications. We present a transform from Boubaker polynomial to Legendre orthogonal polynomials. By the transform, the spectral-type numerical method using Boubaker polynomial can be deduced for seeking numerical solution of differential equations.

\section{The Boubaker Polynomials and Their Properties}

The first definition of Boubaker polynomials[8,14-15] is

$$
B_{n}(x)=\sum_{p=0}^{\xi(n)}\left[\frac{(n-4 p)}{(n-p)} C_{n-p}^{p}\right](-1)^{p} x^{n-2 p}
$$

where $\xi(n)=\lfloor n / 2\rfloor$ is the floor function of $n / 2$ and $C_{n}^{p}$ is the binomial coefficient. To computing the polynomials (1), the following recurrence relationship is valid

$$
\left\{\begin{array}{l}
B_{0}(x)=1, B_{1}(x)=x, B_{2}(x)=x^{2}+2, \\
B_{n}(x)=x B_{n-1}(x)-B_{n-2}(x), \quad n>2 .
\end{array}\right.
$$

The Boubaker polynomials also can be defined through the differential equation

$$
\left(x^{2}-1\right)\left(3 n x^{2}+n-2\right) y^{\prime \prime}+3 x\left(n x^{2}+3 n-2\right) y^{\prime}-n\left(3 n^{2} x^{2}+n^{2}-6 n+8\right) y=0 \text {. }
$$

Some properties of the polynomial (1) (2) or (3) are presented in [6-8]. It is interesting to note that some of the properties are very similar with Chebyshev orthogonal polynomials such as three-term recurrence relationship (2), parity, Christoffel-Darboux formula, etc. Whereas, the polynomials system (1) are essentially distinguished from Chebyshev orthogonal polynomials because they are non-orthogonal associated with any m-distribution.

The special subset of the Boubaker polynomials is the case of $n=4 k$ which is often used for BPES. 


\section{The Boubaker Polynomial Expansion Scheme (BPES) and Its Applications}

The definition of the BPES may be as the following [6]:

For a complex function $f(x)$ of a real argument $x$ defined in the interval $-a<x<a$, a BPES- related weak solution of the equation

$$
f(x)=Z_{0}
$$

where $Z_{0}$ is a given complex number, is obtained by calculating the set of complex coefficient $\xi_{k}, k=1, \ldots, N$, which minimizes the real functional

$$
\Psi=\left|\lim _{N \rightarrow \infty} \frac{1}{2 N} \sum_{k=1}^{N} \xi_{k} B_{4 k}\left(\frac{x}{a} \alpha_{k}\right)-Z_{0}\right| .
$$

The following properties of the $4 k$-order Boubaker polynomial is preferred:

$$
\left[\sum_{k=1}^{N} B_{4 k}\left(\frac{x}{a} \alpha_{k}\right)\right]_{x=0}=-2 N, \quad\left[\sum_{k=1}^{N} B_{4 k}{ }^{\prime}\left(\frac{x}{a} \alpha_{k}\right)\right]_{x=0}=0, \quad\left[\sum_{k=1}^{N} B_{4 k}\left(\frac{x}{a} \alpha_{k}\right)\right]_{x=a}=0,
$$

which cooperate the initial and boundary conditions.

There are lots of applications of BPES [8-14] which touch upon different science fields [13] including heat transfer, nonlinear dynamical system, biology, thermodynamics, mechanics, cryogenics, etc. It is unfortunately that there is no error estimation result about BPES approximation. Obviously, the sub-system of $4 k$ - order Boubaker polynomial is incomplete in sense of square integrable function space.

\section{Boubaker-Legendre Transform}

We deduce a transform from Boubaker to the shifted Legendre polynomials, named BoubakerLegendre. Due to independence of Boubaker polynomial system, a $n$-degree polynomial $p_{n}(x)$ can be rewritten as

$$
p_{n}(x)=\sum_{j=0}^{n} a_{j} B_{j}(x)=\sum_{k=0}^{n} b_{k} L_{k}(x)
$$

where $L_{n}(x)$ is the shifted Legendre orthogonal polynomial of degree $n$ associated interval [0,2] . With notations $A=\left[a_{0}, a_{1}, \ldots, a_{n}\right]^{T}$ and $B=\left[b_{0}, b_{1}, \ldots, b_{n}\right]^{T}$, the Boubaker-Legendre transform is the map from $A$ to $B$.

Multiplying (4) by $L_{i}(x)$ and integrating it over $[-1,1]$ and making use of the orthogonality of Legendre polynomials, we obtain

Denote

$$
\frac{2}{2 i+1} b_{i}=\sum_{j=0}^{n} a_{j} \int_{0}^{2} B_{j}(x) L_{i}(x) d x, \quad i=0,1, \ldots, n .
$$

$$
t_{i j}=\frac{2 i+1}{2} \int_{0}^{2} B_{j}(x) L_{i}(x) d x, \quad T=\left(t_{i j}\right)_{i, j=0, \ldots, n} .
$$

Then we have $B=T A$. By using the three-term recurrence relationship (2) and the parity of Boubaker polynomials, we can compute the entries of $T$ by the following relation:

and initial conditions

$$
t_{i, k}= \begin{cases}\frac{i+1}{2 i+3} t_{i+1, k-1}+\frac{i}{2 i-1} t_{i-1, k-1}-t_{i, k-2}, i>0, k>2, i \leq k, \\ \frac{1}{3} t_{1, k-1}-t_{0, k-2}, & i=0, \\ 0, & i>k \text { or } i+k \text { is odd },\end{cases}
$$

$$
t_{0,0}=1, \quad t_{0,2}=7 / 3, \quad t_{1,1}=1
$$


With aid of the Boubaker-Legendre transform, the Boubaker polynomial expansion-based methods may be converted to the shifted Legendre polynomial expansion-type method which can be easily solved by using the orthogonality of Legendre polynomial system.

\section{Applications to Numerical Solution of Differential Equations}

Numerically solving differential equations by Boubaker polynomial expansion method, it needs commonly the following steps:

(i) expressing the unknown functions by Boubaker polynomial expansion with $N$ coefficients;

(ii) determining the $N$ coefficients using relations governed by differential equation;

(iii) recovering the values of solution by expression.

The Boubaker-Legendre transform may be used in above-mentioned step (ii) to simplify the computation process. As a model example to illustrate this, we consider the second-order differential equation with boundary value problem as:

$$
y^{\prime \prime}(x)+\lambda y(x)=f(x), \quad 0<x<2 ; \quad y(0)=y(2)=0 .
$$

Let the approximate solution to problem (5) be

$$
y_{N}(x)=\sum_{j=0}^{N} c_{j} B_{j}(x) .
$$

Then the spectral tau-type method with Boubaker polynomial is to determine $\left\{c_{j}\right\}_{j=0}^{N}$ such that

$$
y_{N}(0)=\sum_{j=0}^{N} c_{j} B_{j}(0)=0, \quad y_{N}(2)=\sum_{j=0}^{N} c_{j} B_{j}(2)=0
$$

and for $k=0,1, \ldots, N-2$

$$
\sum_{j=0}^{N} c_{j} \int_{0}^{2} B_{j}^{\prime \prime}(x) B_{k}(x) d x+\lambda \sum_{j=0}^{N} c_{j} \int_{0}^{2} B_{j}(x) B_{k}(x) d x=\int_{0}^{2} f(x) B_{k}(x) d x .
$$

Let $C=\left[c_{0}, c_{1}, \ldots, c_{N}\right]^{T}$ be the unknown column vector. The linear system for $\left\{c_{j}\right\}_{j=0}^{N}$ is $A C=B$, where $A=\left(a_{i j}\right)_{0 \leq i, j \leq N}, \quad a_{N-1, j}=B_{j}(0), a_{N, j}=B_{j}(2)$ and

$$
a_{i j}=\int_{0}^{2}\left[B_{j}^{\prime \prime}(x)+\lambda B_{j}(x)\right] B_{i}(x) d x, 0 \leq j \leq N ; 0 \leq i \leq N-2,
$$

and $B=\left(b_{i}\right)_{0 \leq i \leq N}$ is a column vector,

$$
b_{i}=\int_{0}^{2} f(x) B_{i}(x) d x, 0 \leq i \leq N-2, b_{N-1}=b_{N}=0 .
$$

In computation of (6) and (7), we face with three-type integrals,

$$
\begin{aligned}
\int_{0}^{2} B_{j}(x) B_{i}(x) d x & =\sum_{k=0}^{N} B_{j}\left(\eta_{k}\right) B_{i}\left(\eta_{k}\right) \omega_{k}, \\
\int_{0}^{2} B_{j}^{\prime \prime}(x) B_{i}(x) d x & =\sum_{k=0}^{N} B_{j}^{\prime \prime}\left(\eta_{k}\right) B_{i}\left(\eta_{k}\right) \omega_{k}, \\
\int_{0}^{2} f(x) B_{i}(x) d x & \approx \sum_{k=0}^{N} f\left(\eta_{k}\right) B_{i}\left(\eta_{k}\right) \omega_{k},
\end{aligned}
$$

where $\eta_{i}, \omega_{i}(0 \leq i \leq N)$ are the nodes and weights of the shifted Guass-Legendre quadrature on the interval $[0,2]$, respectively.

If we apply the Boubaker-Legendre transform, we have

$$
y_{N}(x)=\sum_{j=0}^{N} d_{j} L_{j}(x), \quad C=T^{-1} D, D=\left[d_{0}, d_{1}, \ldots, d_{N}\right]^{T} .
$$

Hence, the three integrals (8) are the counterpart of the shifted Legendre plolynomials, say

$$
\int_{0}^{2} L_{j}(x) L_{i}(x) d x, \quad \int_{0}^{2} L_{j}^{\prime \prime}(x) L_{i}(x) d x \quad \text { and } \quad \int_{0}^{2} f(x) L_{i}(x) d x .
$$


After $D=\left(d_{i}\right)_{0 \leq i \leq N}$ is obtained, (9) gives the coefficient $\left\{c_{j}\right\}_{j=0}^{N}$ and then the approximate solution.

\section{Summary}

In this paper, we discussed the Boubaker polynomial-related problems: the definitions of the Boubaker polynomials, some properties of the Boubaker polynomials, Boubaker polynomial expansion scheme(BPES), and their applications. We derive a Boubaker-Legendre transform which links a Boubaker polynomial expansion with shifted Legendre polynomial expansion. By applying the Boubaker-Legendre transform, spectral-tau type method for numerically solving differential equation is described.

\section{Acknowledgement}

This work was supported by the National Nature Science Foundation of China under grant No. 11161026 and 11261027.

\section{References}

[1] G. Szegò, Orthogonal Polynomials, AMS Coll. Publ., Providence, 1975.

[2] J. Mason and D. C. Handscomb, Chebyshev Polynomials, Taylor \& Francis, 2002.

[3] J. P. Boyd, Chebyshev and Fourier Spectral Methods, 2nd ed., Dover, Mineola, New York,2001.

[4]J. Shen, T. Tang and L. L. Wang, Spectral Methods: Algorithms, Analysis and Applications, Springer, 2011.

[5]B. -Y. Guo, Spectral Methods and Their Applications, World Scientific, 1998.

[6]T. G. Zhao, L. Naing and W. Y. Yue, Some new features of the Boubaker polynomials expansion scheme BPES, Mathematical Notes, 87(2010) 175-178.

[7]P. Barry, On the connection coefficients of the Chebyshev-Boubaker polynomials, Scientific World Journal, 2013(2013), doi:10.1155/2013/657806.

[8]K. Boubaker, The Boubaker polynomials, a new function class for solving bi-varied second-order differential equations, Far East J. Appl. Math., 31(3)(2008) 299-320.

[9] H. Kocak, A. Yildirim, D. H. Zhang and S. T. Mohyud-Din, The comparative Boubaker polynomial expansion scheme(BPES) and homotopy perturbation method(HPM) for solving a standard nonlinear second-order boundary value problem, Math. Comput. Modelling, 54(1)(2011) 417-422.

[10]A. Belhadj, O. F. Onyango and N. Rozibaeva, Boubaker polynomials expansion scheme-related heat transfer investigation inside keyhole model, J. Thermophysics and Heat Transfer, 23(3)(2009) 639-640.

[11]S. A. Tabatabaei, T. G. Zhao, O. B. Awojoyogbe and F. O. Moses, Cut-off cooling velocity profiling inside a keyhold model using the Boubaker polynomials expansion scheme, Heat Mass Tranfer, 45(2009) 1247-1251.

[12]B. Dubey, T. G. Zhao, M. Jonsson and H. Rahmanov, A solution to the accelerated-predatorsatiety Lotka-Volterra predator-prey problem using Boubaker polynomial expansion scheme, J. Theor. Biology, 264(2010) 154-160.

[13]Information on https://en.wikiversity.org/wiki/Boubaker_Polynomials.

[14]K. Boubaker, On modified Boubaker polynomials: some differential and analytical properties of the new polynomial issued from an attempt for solving bi-varied heat equation, Trends in Applied Sciences Research, 2(2007) 540-544.

[15]H. Labiadh and K. Boubaker, A Sturm-Liouville shaped characteristic differential equation as a guide to establish a quasi-polynomial expression to the Boubaker polynomials, Diff. Eq. Cont. Proc., 2(2007) 117-133. 